\title{
Notas Editoriais
}

\section{AURELIO RIBEIRO LEITE DE OLIVEIRA ${ }^{1}$ e FABRÍCIO SIMEONI DE SOUSA ${ }^{2}$}

Esta edição marca o início dos trabalhos do prof. Aurelio Ribeiro Leite de Oliveira como novo Editor Chefe da revista TEMA, vindo a substituir o prof. Antônio Castelo Filho, que termina seu mandato de dois anos à frente da revista TEMA, e agora se prepara para uma nova etapa de sua carreira como professor titular no ICMC/USP. Fica aqui nossa homenagem à atuação do prof. Castelo, cuja grande contribuição, além das tarefas inerentes ao cargo de Editor Chefe, foi a reorganização do sistema de gerenciamento da revista e solução dos problemas causados pela desatualização do sistema anterior. Ficamos muito gratos pelo seu tempo dedicado à revista, esperamos continuar contando com suas contribuições no futuro.

Esta última edição de 2015 traz sete artigos versando sobre diferentes áreas da Matemática Aplicada e Computacional, como análise de EDP's, avanços na solução de problemas inversos, lógica fuzzy, análise combinatória e redução de dimensionalidade.

O artigo intitulado "Conjugate Gradient Method for the Solution of Inverse Problems: Application in Linear Seismic Tomography” faz aplicação do método clássico de Gradientes Conjugados na solução de problemas inversos decorrentes de Tomografia Sísmica.

Já na área de análise de EDP's, temos a contribuição do artigo intitulado "Remarks on a Nonlinear Wave Equation in a Noncylindrical Domain", que traz novos resultados sobre a existência e unicidade para um problema de valor de contorno com uma equação da onda não linear em domínios não cilíndricos.

Os dois artigos seguintes trazem abordagens diferentes para a mesma aplicação na identificação de danos estruturais. Enquanto o artigo "Identificação de Danos Estruturais utilizando Dados no Domínio do Tempo e Critério D-ótimo" traz uma abordagem de problema inverso e modelagem de elementos finitos, o artigo "Identificação de Danos Empregando um Modelo de Dano Contínuo e o Método de Monte Carlo com Cadeias de Markov" faz uso também de modelagem de elementos finitos, mas empregando método de Monte Carlo com cadeias de Markov.

$\mathrm{Na}$ área de lógica fuzzy o artigo "Aggregating Fuzzy QL- and (S,N)-Subimplications: Conjugate and Dual Constructions" caracteriza $(\mathrm{S}, \mathrm{N})$ e QL-subimplicações nebulosas usando agregação pela mediana.

\footnotetext{
${ }^{1}$ Universidade Estadual de Campinas - UNICAMP, SP, Brasil. E-mail: aurelio@ ime.unicamp.br

${ }^{2}$ Universidade de São Paulo - USP, São Carlos, SP, Brasil. E-mail: fsimeoni@icmc.usp.br
} 
O artigo seguinte: "Parity Indices and Two-Line Matrix Representation for Partitions", da área de combinatória, apresenta novas demonstrações e resultados na teoria de partição utilizando o conceito de paridade no estudo de identidades.

Finalmente, o artigo: "Feature Extraction of Structures in Sea Water Using Self-Organizing Maps and Electromagnetic Waves" desenvolve um algoritmo para redução de dimensionalidade e identificação de estruturas. Aplicações na detecção de objetos subaquáticos em regiões marinhas demonstram o bom desempenho desta abordagem.

Não deixe de citar os artigos da TEMA em seus trabalhos de pesquisa. Isto aumentará a credibilidade de nosso periódico.

Boa leitura. 